\title{
Age-related Changes of Glu/GABA Expression in the Primary Visual Cortex of Cat
}

\author{
DIAO Jian-gang, XU Jin-wang, LI Gu-zhou, TANG Chuan-hong, HUA Tian-miao* \\ (College of Llife Sciences, Anhui Normal University, Wuhu 241000, China)
}

\begin{abstract}
Recent studies show that a reduced effect of inhibitory transmitter system in the visual cortex may underlie aged visual function degradation. Whether excitatory transmitter system changes with age and hence affects intracortical excitation-inhibition balance is not clear. To explore this issue, we used Nissl staining and immunohistochemical methods as well as Image-Pro Express software to examine the density of Nissl-stained neurons, Glutamic acid-immunoreactive (Glu-IR) neurons and $\gamma$-Aminobutyric acid-immunoreactive (GABA-IR) neurons in the primary visual cortex of young adult and aged cats. The results showed that there was no significant difference in the density of Nissl-stained neurons between young and old cats $(P>0.05)$. However, the density of Glu-IR neurons and GABA-IR neurons in the primary visual cortex of aged cats was significantly lower than that of young ones $(P<0.01)$. The ratio between Glu-IR neurons and GABA-IR neurons was significantly increased in old cats compared to that in young adult ones $(P<0.01)$. These results indicated that the effect of excitatory transmitter system in the old visual cortex was increased relative to the inhibitory transmitter system, which might cause an imbalance between cortical excitation and inhibition and might be an important factor mediating the visual function decline during aging.
\end{abstract}

Key words: Glutamic acid (Glu); $\gamma$-Aminobutyric acid (GABA); Balance of excitation and inhibition; Aging; Primary visual cortex; Cat

\section{猫初级视皮层内 Glu/GABA 表达比率的衰老性变化}

\author{
习建刚，徐金旺，李古州，汤传宏，华田苗* \\ (安徽师范大学 生命科学学院, 安徽 芜湖 241000)
}

摘要: 最近的一些研究结果显示, 视皮层内抑制性递质系统作用减弱可能是导致老年性视觉功能衰退的重要 因素。是否皮层内兴奋性递质系统亦伴随衰老而发生改变并影响皮层内神经兴奋与抑制的平衡尚不清楚。为此, 利用 Nissl 染色和免疫组织化学染色方法以及 Image-Pro Express 图像分析软件对青、老年猫初级视皮层(17 区)内 各层神经元密度、兴奋性递质谷氨酸免疫反应阳性(Glu-immunoreactive, Glu-IR) 神经元密度以及抑制性递质 $\gamma$-氨 基丁酸免疫反应阳性( $\gamma$-aminobutyric acid-immunoreactive, GABA-IR) 神经元密度进行了统计分析。结果显示, 青、 老年猫初级视皮层各层神经元密度均没有明显的年龄性差异 $(P>0.05)$; 与青年猫相比, 老年猫初级视皮层 Glu-IR、 GABA-IR 神经元密度均显著减少 $(P<0.01)$, 而 Glu-IR/GABA-IR 神经元密度比率却显著增大 $(P<0.01)$ 。结果提 示, 老年猫初级视皮层内兴奋性递质系统作用相对增强, 而抑制性递质系统的作用相对减弱, 导致皮层内兴奋一 抑制平衡关系失调, 这可能是引起老年个体视觉功能衰退的重要原因之一。

关键词: 谷氨酸; $\gamma$-氨基丁酸; 兴奋-抑制平衡; 衰老; 初级视皮层; 猫

中图分类号：Q423; Q954.67+1 文献标志码：A 文章编号：0254-5853(2009)01-0038-07

Many visual functions of human beings as well as animals degade with age, such as reduction of visual acuity (Rubin et al, 1997; Hu et al, 2000), decrease of contrast sensitivity to visual signal (Elliott et al, 1990;

Received date: 2008-09-27; Accepted date: 2008-12-12

Foundation items: Natural Science Fund of Anhui Province (070413138); Key Laboratory Foundation of Anhui Province for Researches on the Conservation and Utilization of Important Biological Resource; Key Laboratory Foundation for Universities and Colleges in Anhui

收稿日期：2008-09-27; 接受日期：2008-12-12

*通讯作者 (Corresponding author), E-mail: tianmiaohua@gmail.com

第一作者（First author）,E-mail: diaojg2cy@126.com 
Nomura et al, 2003), decline of sensitivity to wavelength (Johnson et al, 1988; Gray et al, 1995) as well as the selectivity to orientation and/or motion direction of visual stimulus (Willis \& Anderson, 2000; Kline et al, 2001). The primary visual cortex receives signal input directly from lateral geneculate nucleus (LGN) and plays a quite important role in the preliminary processing of visual information and relaying processed visual information to higher cortical loci for visual perception (Shou, 2006). Similar to other cerebral cortex, the gray matter of primary visual cortex can be divided into six layers from the pial surface to white matter, including molecular lamina, exeternal granular lamina, external pyamidal lamina, internal granular lamina, internal pyramidal lamina and multiform lamina. The layer IV, which is thicker than other layers, is the main input region of visual information, while the layer II and III are the main region of visual information output to the higher visual cortices, and the layer $\mathrm{V}$ and VI export feedback signals to subcortical nucleus, including superior colliculus (SC) and lateral geneculate nucleus (LGN) (Shou, 2006). Therefore, age-related morphological or functional changes in the primary visual cortex may exert a great impact on various visual functions.

Recent studies show that a reduction of inhibitory transmitter system effect in the visual cortex may mediate aged visual function degradation (Schmolesky et al, 2000; Leventhal et al, 2003; Hua et al, 2006, 2008). Whether excitatory transmitter system modifies with age and thus impacts intracortical excitation-inhibition balance remains unknown. To probe into this issue, we used Nissl staining and immunohistochemical methods as well as Image-Pro Express software to examine the density of Nissl-stained neurons, Glu-IR and GABA-IR neurons in the primary visual cortex of young adult and aged cats, attempting to explore if the excitatory transmitter system changes during senescence and provide morphological evidence for further researches on mechanisms of visual function decline during aging.

\section{Material and methods}

\subsection{Preparation of subjects and tissue}

Four young adult cats (1-3 years old) and 4 old cats (11-13 years old), all of which were healthy cats and examined ophthalmoscopically before the experiment to confirm that there is no optical or retinal problem that would impair their visual function, were studied in this experiment. All the animals were treated strictly in accordance with the National Institutes of Health Guide for the Care and Use of Laboratory Animals.

The animals were deeply anaesthetized with ketamine hydrochloride $(40 \mathrm{mg} / \mathrm{kg})$ and then perfused with $0.9 \% \mathrm{NaCl}$ solution through the ascending aorta till the liver became pale, followed immediately by $500 \mathrm{~mL}$ of a fixative solution containing 4\% Paraformaldehyde and $2.5 \%$ Glutaraldehyde in $0.1 \mathrm{~mol} / \mathrm{L}$ phosphate buffer solution (PBS, pH7.2-7.4). After perfusion, the skull was opened and the blocks of tissue containing $V_{1}$ were dissected out and cut into about $1 \mathrm{~cm}$ in length. After further fixation for $20-24 \mathrm{~h}$ in the perfusing fixative solution, the samples were washed in PBS $(3 \times 5 \mathrm{~min})$, dehydrated in ethanol, transparentized in xylene and embedded in paraffin. Consecutive coronal sections of 7 $\mu \mathrm{m}$ thick were cut perpendicularly to the pial surface of the lateral gyrus in V1 and then dried onto APES-coated glass slides at $37^{\circ} \mathrm{C}$ for $2-3$ days. Every other 10 slices, 4 adjacent ones were choosed and used respectively for Nissl staining, immunohistochemical labeling of Glu and GABA neurons as well as negative control experiment.

\subsection{Procedure of immunohistochemical and Nissl staining}

Paraffin slices used for immunostaining were first washed in $0.1 \mathrm{~mol} / \mathrm{L}$ PBS (pH7.2-7.4) for $10 \mathrm{~min}$ after deparaffinizing in xylene and rehydrating in gradient ethanol, then incubated with $0.3 \% \mathrm{H}_{2} \mathrm{O}_{2}$ in PBS $(3 \times 5$ $\mathrm{min})$ to quench endogenous peroxidase activity. Following washed in PBS $(3 \times 5 \mathrm{~min})$, the slices were incubated with 5\% normal goat serum in PBS for $10 \mathrm{~min}$ at room temperature to block non-specific reactions. Subsequently, the slices were incubated respectively with primary antibody against Glu and GABA for $24 \mathrm{~h}$ at $4{ }^{\circ} \mathrm{C}$, washed in PBS $(3 \times 5 \mathrm{~min})$ and then incubated with biotinylated rabbit anti-cat $\operatorname{IgG}$ for $15-20 \mathrm{~min}$ at $37^{\circ} \mathrm{C}$. After further rinsing in PBS $(3 \times 5 \mathrm{~min})$, the slices were incubated with an $\mathrm{ABC}$ solution for 15-20 min. After rinsing in $\mathrm{PBS}(3 \times 5 \mathrm{~min})$, dehydrating in gradient alcohol and clearing in xylene, the slices were finally coverslipped with permont. Slice used for control experiment was stained simultaneously following the same procedure as described above with the exception that the primary antibody was omitted. The paraffin slice used for Nissl staining was put into $0.25 \%$ cresyl violet solution for $1-2 \mathrm{~h}$ at room temperature after deparaffinizing and rehydrating, then dehydrated, cleared and finally coverslipped. Nissl staining was used for 
identification of cortical layers and statistical evaluation of the density of total cortical neurons in each layer of the primary visual cortex.

The dyestuff of cresyl violet used in this experiment was bought from the corporation of Sigma. The primary antibodies against Glu and GABA, the reagent box for immunohistochemistry and the DAB colored fluid were all bought from the company of Lab Vision Corporation.

\subsection{Statistical analysis}

Under an Olympus BX51 microscope, statistical studies were performed at the medial bank of the lateral gyrus (V1) where the cortical surface runs approximately parallel to the white matter. In each Nissl-stained section, the mean depth of each cortical layer was measured with an optical calibrator at a low magnification $(\times 4$ or $\times 10$ objective lens with $\times 10$ eyepieces) and used for laminar localization in cell counting. At a high magnification $(X$ 40 objective lens with $\times 10$ eyepieces), the number of total cortical neurons, Glu-IR neurons and GABA-IR neurons within varied cortical layers (layer I, II -III, $\mathrm{IV}$ and $\mathrm{V}-\mathrm{VI}$ ) were randomly counted for 6 visual fields in each section using a grid of counting frame (50 $\mu \mathrm{m} \times 50 \mu \mathrm{m})$. For an unbiased counting, neurons whose nuclear profile intersected the right and upper border of the frame were included for analysis while those crossing the left and lower border were excluded. The density of Nissl-stained neurons, Glu-IR neurons and GABA-IR neurons in different cortical layer was obtained in each

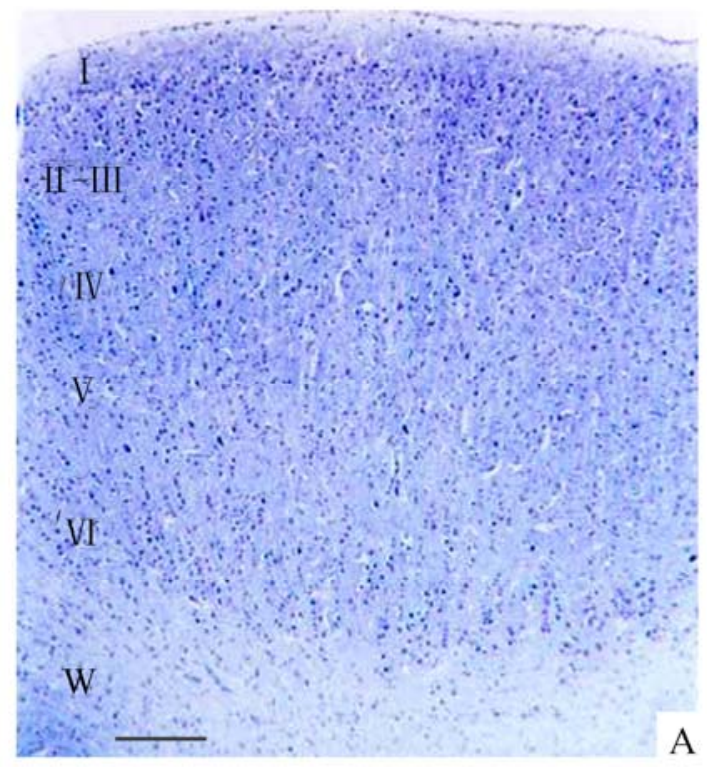

Nissl-stained and immuno-stained section, and the ratios of Glu-IR neurons to GABA-IR neurons in different cortical layers were acquired for each adjoining section group. The criteria for acceptance as a neuron in Nissl-stained section were a clear differentiation from background staining of a soma and the presence of a nucleus. A cell with a nucleus and a brown or dark brown-labeled soma that clearly distinguished from background staining was designated as a Glutamate- or GABA-positive neuron in the immunostaining sections.

All the data were expressed as mean \pm standard deviation. The significance of the differences between cortical layers, individuals and age groups was evaluated by one- or two-way analysis of variance (ANOVA), and a difference with $P$-value of $<0.05$ was accepted as statistical significant.

\section{Results}

\subsection{Result of Nissl staining}

In Nissl-stained sections, the gray matter of primary visual cortex of cat can be devided into 6 layers from the outside to the inside (Fig. 1), including molecular lamina ( I ), exeternal granular-pyamidal lamina( II - III ), internal granular lamina ( IV ), internal pyramidal lamina(V) and multiform (VI). Our statistical analysis indicated that the density of Nissl-stained neurons in each cortical layer showed no significant difference between individuals of either young adult cat group

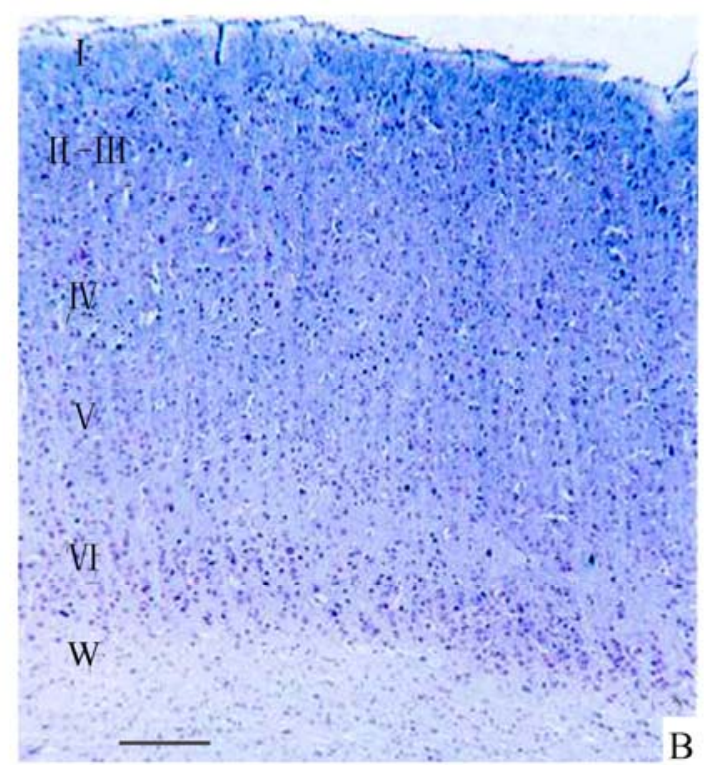

Fig. 1 Nissl staining shows the laminar identification in primary visual cotex of young adult and aged cats Layer identification of primary visual cortex in young cats (A) and aged cats (B)

I, II-III, IV, V and VI represents morlecular laminar, exetermal granular-pyamidal laminar, internal granular laminar, internal pyramidal laminar and multiform laminar of gray matter of primary visual cortex respectively, W represents white matter. Bar $=300 \mu \mathrm{m}$, the dyestuff used is cresyl violet. 
Tab. 1 Density of neurons of the primary visual cortex in young and old cats $(M e a n \pm S D)$

\begin{tabular}{lcc}
\hline \multicolumn{1}{c}{ Neurons } & Young cats $\left(\right.$ cells $\left./ \mathrm{mm}^{2}\right)$ & Old cats $\left(\right.$ cells $\left./ \mathrm{mm}^{2}\right)$ \\
\hline Neurons in layer I & $268.07 \pm 48.42$ & $259.61 \pm 34.67$ \\
Neurons in layer II -III & $1142.81 \pm 162.30$ & $1127.53 \pm 145.32$ \\
Neurons in layer IV & $1134.63 \pm 150.21$ & $1115.72 \pm 129.74$ \\
Neurons in layer V-VI & $1087.29 \pm 121.56$ & $1063.14 \pm 117.83$ \\
\hline
\end{tabular}

(main effect of cat: $F_{(3,396)}=0.197, P=0.898$ ) or old cat one [main effect of cat: $\left.F_{(3,396)}=0.164, P=0.921\right]$. Between-group analysis neither revealed age difference in the density of Nissl-stained neurons of all cortical layers [main effect of age: $F_{(1,792)}=2.349, P=0.126$ ] (Tab. 1).

\subsection{Result of Glu and GABA immunostaining}

In immunostaining sections, Glu-IR neurons and GABA-IR neurons were found throughout all layers of $\mathrm{V} 1$ both in young adult and old cats as indicated by the presence of brown or dark brown-stained cell bodies (Fig. $2 \mathrm{~A}-\mathrm{G}$ ). A number of Glu-IR and GABA-IR neurons in $\mathrm{V} 1$ of young cats exhibited a clear labeling of the proximal dendrites and/or an initial segment of the axons (Fig. 2A, C, E, G), but very few neuron in old cats showed any clear dendrites or axons (Fig. 2B, D, F, H). Compared with young adult cats, the Glu-IR neurons in $\mathrm{V}_{1}$ of old cats was evidently sparser (Fig. 2A, B, C, D). Similarly, the GABA-IR neurons in $\mathrm{V}_{1}$ of old cats was also less than that in young adult cats (Fig. 2E, F, G, H). There was no positive reaction in the negative control experiment.

2.2.1 Density of Glu-IR and GABA-IR neurons The statistical result indicated that both density of Glu-IR neurons and GABA-IR neurons in each cortical layer of $\mathrm{V}_{1}$ showed no significant difference within either the young adult cat group [Glu: $F_{(3,396)}=0.198, P=0.897$; GABA: $F_{(3,396)}=0.604, P=0.613$ ] or the old cat group
[Glu: $F_{(3,396)}=0.174, P=0.914$; GABA: $F_{(3,396)}=0.686$, $P=0.561]$. However, inter-group comparisons showed that both density of Glu-IR neurons and GABA-IR neurons had significant age difference [Glu: $F_{(1,792)}=$ 7.343, $P=0.007$; GABA: $\left.F_{(1,792)}=478.213, \quad P<0.0001\right]$ (Tab. 2). Compared with young adult cats, the mean density of Glu-IR neurons in old cats decreased respectively by $12.8 \%, 15.0 \%, 14.3 \%$ and $9.5 \%$ in layer I, II-III, IV, and V-VI; while the mean density of GABA-IR neurons reduced by $45.7 \%, 42.8 \%, 43.4 \%$ and $47.9 \%$ in layer I, II-III, IV and V-VI respectively. 2.2.2 Ratios of Glu-IR to GABA-IR neuron density

Our statistical result showed that the ratios of Glu-IR neurons to GABA-IR neurons in the primary visual cortex of old cat were significantly higher than that of young adult cat [main effect of age: $F_{(1,792)}=960.034$, $P<0.0001]$. Relative to young adult cats, the mean ratios of Glu-IR neurons to GABA-IR neurons in old ones increased by $46.1 \%, 64.5 \%, 61.7 \%$ and $60.8 \%$ in layer I, II-III, IV and V-VI respectively (Fig. 3).

In summary, the density of total neurons in primary visual cortex of aged cats showed no significant difference from that of young adult ones. Relative to young adult cats, both the density of Glu-IR neurons and GABA-IR neurons in the primary visual cortex of aged cats decreased to a different extent, but the ratios of Glu-IR neurons to GABA-IR neurons was increased remarkably.

Tab. 2 Density of Glu-IR and GABA-IR neurons in the primary visual cortex of young and old cats $(M e a n \pm S D)$

\begin{tabular}{lll}
\hline \multicolumn{1}{c}{ Neurons } & Young cats $\left(\right.$ cells $\left./ \mathrm{mm}^{2}\right)$ & Old cats $\left(\right.$ cells $\left./ \mathrm{mm}^{2}\right)$ \\
\hline Glu-IR neurons in layer I & $178.63 \pm 33.55$ & $155.76 \pm 25.28^{*}$ \\
Glu-IR neurons in layer II - III & $822.12 \pm 67.97$ & $699.07 \pm 41.05^{*}$ \\
Glu-IR neurons in layer IV & $794.24 \pm 74.43$ & $680.59 \pm 58.26^{*}$ \\
Glu-IR neurons in layer V-VI & $717.61 \pm 58.32$ & $649.52 \pm 46.31^{*}$ \\
GABA-IR neurons in layer I & $69.73 \pm 8.04$ & $37.84 \pm 5.54^{*}$ \\
GABA-IR neurons in layer II-III & $235.58 \pm 45.78$ & $134.87 \pm 21.26^{*}$ \\
GABA-IR neurons in layer IV & $228.16 \pm 43.90$ & $129.42 \pm 29.15^{*}$ \\
GABA-IR neurons in layer V-VI & $216.88 \pm 37.59$ & $113.63 \pm 23.07^{*}$ \\
\hline
\end{tabular}

${ }^{*}$ Means significant difference between young and old cats, $P<0.01$. 

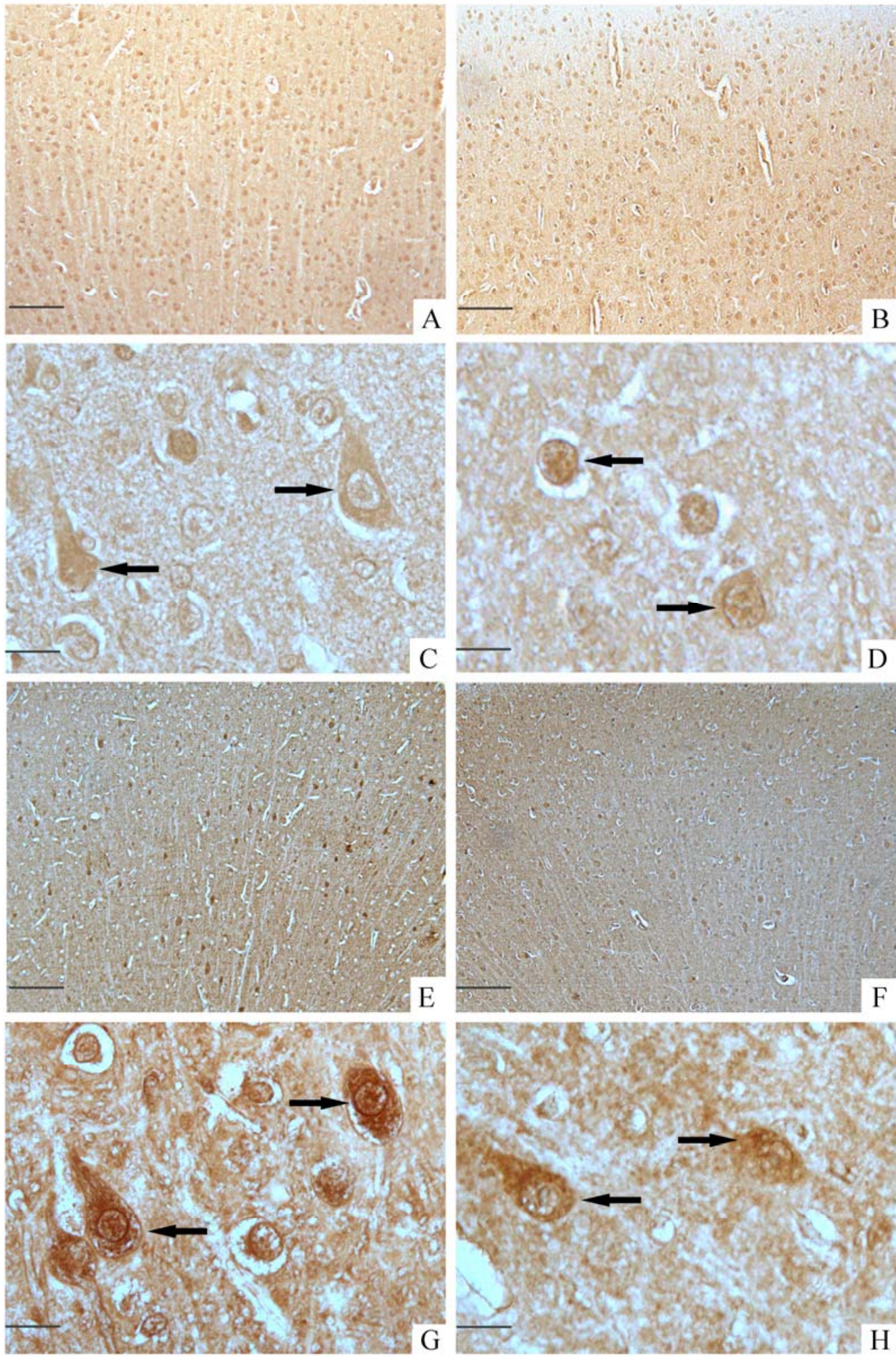

Fig. 2 Immunohistochemical staining shows Glu-IR and GABA-IR neurons in V1 of young adult and aged cats

Glu-IR neurons in layer II -III, IV of primary visual cortex in young cats (A) and aged cats (B), Bar=200 $\mu \mathrm{m}$; Glu-IR neurons $(\rightarrow)$ in layer IV of primary visual cortex in young cats (C) and aged cats (D), Bar=25 $\mu \mathrm{m}$; GABA-IR neurons in layer II -III, IV of primary visual cortex in young cats (E) and aged cats (F), Bar=200 $\mu \mathrm{m}$; GABA-IR neurons $(\rightarrow)$ in layer IV of primary visual cortex in young cats $(\mathrm{G})$ and aged cats $(\mathrm{H}), \mathrm{Bar}=25 \mu \mathrm{m}$. 


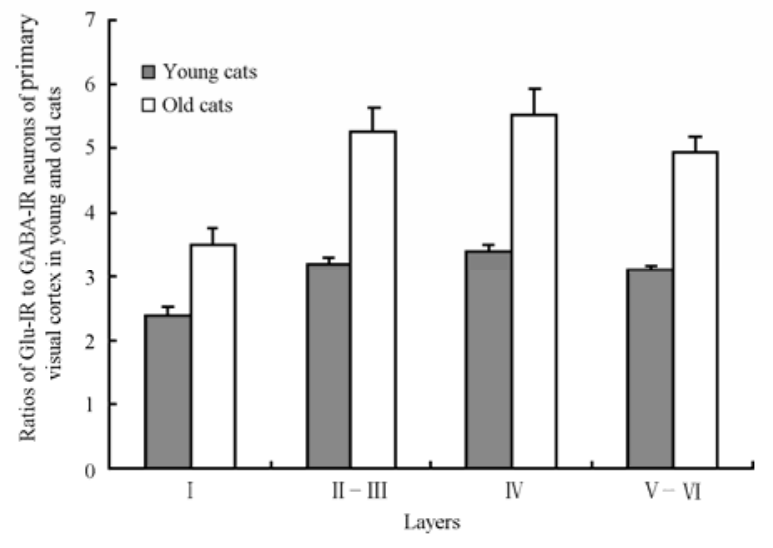

Fig. 3 Ratios of Glu-IR to GABA-IR neurons in different layers of the primary visual cortex in young and old cats

\section{Discussion}

In the transmiting process of visual signal, the physiological function of primary visual cortex is mainly to receive the visual information (such as visual stimulus orientation, motion direction and contrast sensitivity) from LGN and relay it to higher visual cortex after preliminary information processing. A number of researches indicated that human beings and animals showed lower capacity for visual stimulus orientation and motion direction discrimination during aging. For example, aged people learned very slowly in tasks related to orientation and motion direction (Owsley et al, 1981; Tran et al, 1998), and the ability to detect moving signals also dropped down significantly with age (Willis \& Anderson, 2000; Kline et al, 2001). Recent studies showed that a reduced effect of inhibitory transmitter system in the visual cortex may underlie aged visual function degradation. $\mathrm{Hu}$ et al (2000) found that iontophoretic application of $\mathrm{GABA}_{\mathrm{A}}$ receptors antagonists into the primary visual cortex of cat could obviously depress the selectivity of LGN neurons to visual stimuli orientations. Schmolesky et al (2000) suggested that a compromised effect of inhibitory transmitter system in aged monkeys' cortex could account for the decreased selectivity of $\mathrm{V}_{1}$ neurons for visual stimulus properties, which was largely attributed to an increase of spontaneous activity and response to all visual stimulus orientations and motion directions, especially to non-optimal orientations and motion directions. Leventhal et al (2003) found that adding GABA or the agonist of GABA receptors to the visual cortex of old monkey could greatly improve the selectivity of visual cortical neurons for stimulus orientations and motion directions. Our previous studies using extracellular single-unit recording techniques showed that the neurons in primary visual cortex of old cats exhibited degraded functions as indicated by a lower signal-to-noise ratio, higher spontaneous activity, less selectivity to orientations and motion directions of visual stimulus than did neurons of young adult cats (Hua et al, 2006), and our recent studies indicated that this declined stimulus selectivity of visual cortical neurons in the aged animals may attribute to the reduced synthesis of inhibitory neurontransmitter in the aged cortex (Hua et al, 2008). In current study, we compared the density of Glu-IR and GABA-IR neurons as well as the ratio of Glu-IR neuron density to GABA-IR neuron density in the primary visual cortex between aged and young adult cats. We found that both the density of Glu-IR neurons and GABA-IR neurons in the primary visual cortex of old cats were significantly reduced. However, the ratio of Glu-IR neurons to GABA-IR neurons in old cats was signifcantly higher than that in young adults. Therefore, the effect of excitatory transmitter system in the primary visual cortex of old cats was actually enhanced relative to the inhibitory transmitter one.

Glutamate and GABA is the most important excitatory and inhibitory neurontransmitter in the central nervous system. Excitatory input connections are capable of generating the selectivity of visual cortical neurons to stimulus orientations and motion directions while intracortical inhibitory connections tend to suppress the neuronal response, especially the response to visual stimuli with non-optimal orientations and motion directions, and thus sharpen its stimulus tuning selectivity (Sato et al, 1996; Crook et al, 1996, 1997, 1998; Sohya et al, 2007), so the balance between intracotical excitation and inhibition will play a key role in keeping the normal brain function. An imbalance between cortical excitation and inhibition, which was highlighted by lots of researchers, has been deemed as an important reason for brain damnification (Lyden \& Hedges, 1992; Shuaib \& Kanthan, 1997; Green et al, 2000; Schwartz-Bloom \& Sah, 2001). Further experimental evidences indicated that intracortical inhibitory connections experience spatial pattern refining and clustering during postnatal development and remain spatially aligned with excitatory connections in the young and probably middle-aged adulthood so that visually evoked excitatory inputs are selectively masked 
and hence keep neurons responding sharply to optimal orientations and motion directions (Chen et al, 2005; Marino et al, 2005). In this study, both the density of Glu-IR neurons and GABA-IR neurons in primary visual cortex of old cats reduced. However, a varied decrease rate of Glu-IR and GABA-IR neurons led to an increased ratio of Glu-IR nuerons to GABA-IR neurons in the primary visual cortex of old cats relative to young adults.

To sum up, the expression of excitatory neurontransmitter is enhanced relative to the inhibitory

\section{References:}

Chen B, Boukamel K, Kao JP, Roerig B. 2005. Spatial distribution of inhibitory synaptic connections during development of ferret primary visual cortex [J]. Brain Res, 160(4): 496-509.

Crook JM, Kisvarday ZF, Eysel UT. 1996. GABA-induced inactivation of functionally characterized sites in cat visual cortex (area 18): Effects on direction selectivity [J]. J Neurophysiol, 75(5): 2071-2088.

Crook JM, Kisvarday ZF, Eysel UT. 1997. GABA-induced inactivation of functionally characterized sites in cat striate cortex: effects on orientation tuning and direction selectivity [J]. Vis Neurosci, 14(1): 141-158.

Crook JM, Kisvarday ZF, Eysel UT. 1998. Evidence for a contribution of lateral inhibition to orientation tuning and direction selectivity in cat visual cortex: Reversible inactivation of functionally characterized sites combined with neuroanatomical tracing techniques [J]. Eur J Neurosci, 10(6): 2056-2075.

Elliott D, Whitaker D, MacVeigh D. 1990. Neural contribution to spatiotemporal contrast sensitivity decline in healthy ageing eyes $[\mathrm{J}]$. Vision Res, 30(4): 541-547.

Green AR, Hainsworth H, Jackson DM. 2000. GABA potentiation: a logical approach for the treatment of acute ischaemi stroke [J]. Neuropharmacology, 39(9): 1483-1494.

Gray LS, Heron G, Cassidy D, Clark GM, Cowley GR, Gourlay DM, Ross FM. 1995. Comparison of age-related changes in short wavelength sensitive cone thresholds between normals and patients with primary open angle glaucoma [J]. Optom Vis Sci, 72(3): 205-209.

Hu B, Li X, Zhou Y, Shou T. 2000. Effects of bicuculline on direction-sensitive relay cells in the dorsal lateral geniculate nucleus (LGNd) of cats [J]. Brain Res, 885(1): 87-93.

Hua T, Li X, He L, Zhou Y, Wang Y, Leventhal AG. 2006. Functional degradation of visual cortical cells in old cats [J]. Neurobiology of Aging, 27(1): 155-162.

Hua T, Kao C, Sun Q, Li X, Zhou Y. 2008. Decreased proportion of GABA neurons accompanies age-related degradation of neuronal function in cat striate cortex [J]. Brain Research Bulletin, 75(1): 119-125.

Johnson CA, Adams AJ, Twelker JD, Quigg JM. 1988. Age-related changes in the central visual field for short wavelength sensitive pathways $[J]$. $J$ Opt Soc Am A, 5(12): 2131-2139.

Kline DW, Culham JC, Bartel P, Lynk L. 2001. Aging effects on vernier hyperacuity: A function of oscillation rate but not target contrast [J]. Optom Vis Sci, 78(9): 676-682.

Leventhal AG, Wang Y, Pu M, Zhou Y, Ma Y. 2003. GABA and its agonists improved visual cortical function in senescent monkeys [J]. Science, one in the primary visual cortex of cats during aging and hence result in a malajustment of excitation-inhibition balance, which may be an important reason for the decline of visual function in aged individuals. Of course, expression of other neurontransmitter such as acetylcholine, noradrenalin, dopamine as well as glycin may also change with age and hence affect the intracortical excitation-inhibition balance, which need to be studied subsequently.

300(5620): 812-815.

Lyden PD, Hedges B. 1992. Protective effect of synapatic inhibition in cerebral ischemia in rats and rabbits [J]. Stroke, 23(10): 1463-1470.

Marino J, Schummers J, Lyon DC, Schwabe L, Beck O, Wiesing P, Obermayer K. 2005. Invariant computations in local cortical networks with balanced excitation and inhibition [J]. Nat Neurosci, 8(2): 194-201.

Nomura H, Ando F, Niino N, Shimokata H, Miyake Y. 2003. Age-related change in contrast sensitivity among Japanese adults [J]. Jpn $J$ Ophthalmol, 47(3): 299-303.

Owsley C, Sekuler R, Boldt C. 1981. Aging and low-contrast vision: Face perception [J]. Invest Ophthalmol Vis Sci, 21(2): 362-365.

Rubin GS, West SK, Munoz B, Bandeen-Roche K, Zeger S, Schein O, Fried LP. 1997. A comprehensive assessment of visual impairment in a population of older Americans. The SEE study. Salisbury eye evaluation project [J]. Invest Ophthalmol Vis Sci, 38(3): 557-568.

Sato H, Katsuyama N, Tamura H, Hata Y, Tsumoto T. 1996. Mechanisms underlying orientation selectivity of neurons in the primary visual cortex of the macaque [J]. J Physiol, 494(3): 757-771.

Shou TD. 2006. Neurobiology[M]. Beijing: Higher Education Press, 207-221.

Schmolesky MT, Wang Y, Pu M, Leventhal AG. 2000. Degradation of stimulus selectivity of visual cortical cells in senescent rhesus monkeys [J]. Nat Neurosci, 3(4): 384-390.

Schwartz-Bloom RD, Sah R. 2001. $\gamma$-Aminobutyric acid (A) neurotransmission and cerebral ischemia $[\mathrm{J}]$. J Neurochem, 77(2): 353-371.

Shuaib A, Kanthan R. 1997. Amplification of inhibitory mechanisms in cerebral ischemia: An alternative approach to neuronal protection $[\mathrm{J}]$. Histol Histopathol, 12 (1): 185-194.

Sohya K, Kameyama K, Yanagawa Y, Obata K, Tsumoto T. 2007. GABAergic neurons are less selective to stimulus orientation than excitatory neurons in layer II/III of visual cortex, as revealed by in vivo functional $\mathrm{Ca}^{2+}$ imaging in transgenic mice [J]. J Neurosci, 27(8): 2145-2149.

Tran DB, Silverman SE, Zimmerman K, Feldon SE. 1998. Age-related deterioration of motion perception and detection [J]. Graefes Arch Clin Exp Ophthalmol, 236(4): 269-273.

Willis A, Anderson SJ. 2000. Effects of glaucoma and aging on photopic and scotopic motion perception [J]. Invest Ophthalmol Vis Sci, 41(1): 325-335. 